\title{
Mechanisms of Liquid Stream Breakup: Vortices and Time and Length Scales
}

\author{
A. Zandian ${ }^{1}$, W. A. Sirignano*1, F. Hussain ${ }^{2}$ \\ ${ }^{1}$ Department of Mechanical and Aerospace Engineering, University of California, Irvine, USA \\ ${ }^{2}$ Department of Mechanical Engineering, Texas Tech University, Lubbock, USA \\ *Corresponding author: sirignan@uci.edu
}

\begin{abstract}
The 3D, temporal instabilities on a planar liquid jet are studied using DNS with level-set and VoF interface-capturing methods. The $\lambda_{2}$ method has been used to relate the vortex dynamics to the surface dynamics at different stages of the jet breakup. The breakup character depends on the Ohnesorge number $(O h)$ and gas-to-liquid density ratio. At high Reynolds number $(R e)$ and high $O h$, hairpin vortices form on the braid and overlap with the lobe hairpins, thinning the lobes, which then puncture creating holes and bridges. The bridges break, creating ligaments that stretch and break into droplets by capillary action. At low $O h$ and high $R e$, lobe stretching and thinning is hindered by high surface tension and splitting of the original Kelvin-Helmholtz vortex, preventing early hole formation. Corrugations form on the lobe edges, influenced by the split vortices, and stretch to form ligaments. Both mechanisms are present in a transitional region in the $W e$-Re map. At lower $R e$ and not-too-large Weber number $(W e)$, lobe stretching occurs but with longer and larger ligaments in this third domain which has a hyperbolic transition to the hole formation domain as $W e$ increases. The three domains with differing breakup behaviors each occupy distinct portions of a plot of $W e$ based on gas density versus $R e$ based on liquid properties. Characteristic times for the hole formation, as well as the lobe and ligament stretching are different - the former depending on the surface tension and the latter on liquid viscosity. In the transitional region, both times are of the same order.
\end{abstract}

\section{Keywords}

Gas/liquid flow, primary atomization, breakup mechanism, hydrodynamic instability, vortex dynamics.

\section{Introduction}

Earlier computational works on the breakup of liquid streams at higher Weber number $(W e)$ and Reynolds number $(R e)$ focused on the surface dynamics using either volume-of-fluid or level-set methods [1-3]. More recently, Jarrahbashi and Sirignano [4] and Jarrahbashi et al. [5] numerically simulated the temporal behavior of round jets with additional data analysis that related the vorticity dynamics to the surface dynamics. Jarrahbahsi et al. [5] showed that important spray characteristics, e.g. droplet size and spray angle, differed in different ranges of $W e, R e$, and density ratio. Therefore, further studies of the breakup mechanisms are needed to fully understand the causes of these differences. Consequently, there are remaining questions to be addressed in this paper: What are the details of the liquid dynamics in each breakup domain? What causes the difference in the breakup cascade? What are the roles of surface tension, liquid viscosity, and gas density? The answers to these questions would be crucial in understanding and controlling the droplet size distribution in primary atomization of liquid jets.

Vortex dynamics concepts can clearly explain surface deformation of a liquid jet in the primary atomization process. The Kelvin-Helmholtz $(\mathrm{KH})$ instability promotes the formation of spanwise vorticity waves growing into coherent vortices. These vortices evolve into hairpins with counter-rotating streamwise vortices [6]. The streamwise and spanwise vortical waves combine to produce different surface structures, e.g. lobes, bridges, and ligaments, which eventually break up into droplets. The link between the vortex dynamics and surface dynamics in primary atomization is important, but rarely explored and poorly understood, and this study is an attempt to fill that gap.

There have been several studies in the field of jet instabilities from the vortex dynamics perspective. Most of them however do not consider density and viscosity discontinuities. The main focus of these studies has been on understanding and relating vortex stretching [7], vortex tilting [8], and baroclinic effects [9] to the appearance of three-dimensionality in liquid jet instabilities. Earlier experimental studies in this field [6, 8-14] have been followed and reproduced in more detail by numerical computations [1, 4, 5, 15-21].

In the first studies of the role of streamwise vorticity for round liquid jets flowing into a gas, Jarrahbashi and Sirignano [4] and Jarrahbashi et al. [5] showed how the mechanisms of the formation of lobes and ligaments are related to the growth of streamwise vorticity. For control of spray character, it is very valuable to understand the details in the cascade processes following liquid injection for each of the various atomization domains that have been identified [5]. While we do not address control and optimization in this study, it gives justification for the detailed exploration and the behavioral characterizations reported here.

Our objectives for the planar jet are to explain (i) cascade of structures on the liquid surface with time, including lobe, ligament, and droplet formations; (ii) the breakup mechanisms at different flow conditions; (iii) proper definition of the time scale of each of the breakup mechanisms, which would help predict the dominant mechanism at different flow conditions; and (iv) the mechanisms for surface deformation and breakup in different domains using more sophisticated data analysis for the vortex dynamics (i.e. the $\lambda_{2}$ method). 


\section{Numerical Modeling}

The three-dimensional Navier-Stokes (NS) with level-set (LS) and volume-of-fluid (VoF) interface-capturing methods yield computational results for the liquid-segment which captures the liquid-gas interface deformations with time. The incompressible continuity and Navier-Stokes equations follow:

$$
\nabla \cdot \mathbf{u}=0, \quad \frac{\partial(\rho \mathbf{u})}{\partial t}+\nabla \cdot(\rho \mathbf{u u})=-\nabla p+\nabla \cdot(2 \mu \mathbf{D})-\sigma \kappa \delta(d) \overrightarrow{\mathbf{n}},
$$

where $\mathbf{D}$ is the rate of deformation tensor, and $\mathbf{u}$ is the velocity field; $p, \rho$, and $\mu$ are the pressure, density and dynamic viscosity of the fluid, respectively. The last term in the NS equation is the surface tension force per unit volume, where $\sigma$ is the surface tension coefficient, $\kappa$ is the surface curvature, $\delta(d)$ is the Dirac delta function and $\overrightarrow{\mathbf{n}}$ is the unit vector normal to the liquid/gas interface.

Direct numerical simulation is done by using an unsteady three-dimensional finite-volume solver for the NS equations for the planar incompressible liquid sheet segment (initially stagnant), which is subject to instabilities due to a gas stream that flows past it on both sides. A uniform staggered grid is used with the mesh size of $2.5 \mu \mathrm{m}$ and a time step of $5 \mathrm{~ns}$. Third-order accurate QUICK scheme is used for spatial discretization and the Crank-Nicolson scheme for time marching. The continuity and momentum equations are coupled through the SIMPLE algorithm. The LS method developed by Osher and his coworkers [23-25] captures the liquid-gas interface. The level set $\phi$ is a distance function which is advected by the velocity field:

$$
\frac{\partial \phi}{\partial t}+\mathbf{u} \cdot \nabla \phi=0
$$

For detailed descriptions for this interface capturing see [24].

At low density ratios, a transport equation similar to equation (2) is used for the volume fraction $f$, also called the VoF-variable, in order to describe the temporal and spatial evolution of the two-phase flow [26]. The VoF-variable $f$, introduced by Hirt and Nichols [26], represents the volume of (liquid phase) fluid fraction at each cell.

The computational domain, shown in Figure 1, consists of a cube, which is discretized into uniform-sized cells. The liquid segment, which is a sheet of thickness $h(h=200 \mu \mathrm{m})$ is located at the center of the box and is stationary in the beginning. The domain size in terms of the sheet thickness is $4 h \times 4 h \times 8 h$, in the $x, y$ and $z$ directions, respectively. The liquid segment is surrounded by the gas zones on top and bottom. The gas moves in the positive $x$-direction (streamwise direction), with a constant velocity $(U=100 \mathrm{~m} / \mathrm{s})$ at the top and bottom boundaries, and its velocity diminishes to the interface velocity with a hyperbolic tangent profile. The velocity decays exponentially to zero at the center of the liquid sheet. For more detailed description of the initial conditions, see Zandian et al. [22] Grid independency and domain-size independency tests have also been performed [4, 5, 22].

The liquid-gas interface is initially perturbed symmetrically on both sides with a sinusoidal profile and predefined wavelength and amplitude obtained from 2D full-jet simulations [22]. Both streamwise ( $x$-direction) and spanwise ( $y$-direction) perturbations are considered in this study. Periodic boundary condition for all components of velocity as well as the level-set/VoF variable is imposed on the four sides of the computational domain.

The most important dimensionless groupings in this study are the Reynolds number $(R e)$, the Weber number $(W e)$, density ratio $(\hat{\rho})$, viscosity ratio $(\hat{\mu})$, and the initial wavelength-to-sheet-thickness ratio $(\Lambda)$, as defined below.

$$
R e=\frac{\rho_{l} U h}{\mu_{l}}, \quad W e=\frac{\rho_{l} U^{2} h}{\sigma}, \quad \hat{\rho}=\frac{\rho_{g}}{\rho_{l}}, \quad \hat{\mu}=\frac{\mu_{g}}{\mu_{l}}, \quad \Lambda=\frac{\lambda}{h} .
$$

The sheet thickness $h$ and the velocity of the far field gas $U$ are considered as the characteristic length and velocity. The subscripts $l$ and $g$ refer to the liquid and gas, respectively.

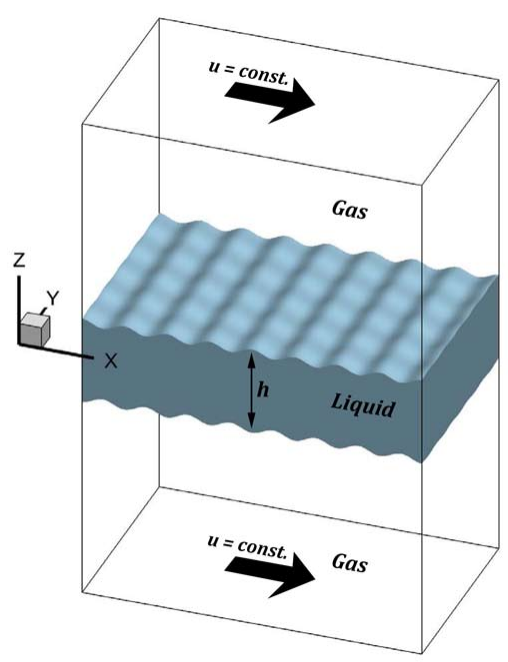

Figure 1. The computational domain with the initial liquid and gas zones. 
Our goal is to study the vorticity dynamics as well as the liquid surface dynamics in order to understand breakup mechanisms at different flow conditions. To this end, $\lambda_{2}$ criterion introduced by Jeong and Hussain [27] is used to define a vortex.

\section{Results and discussion}

Three mechanisms for liquid sheet surface deformation and breakup are identified, which are well categorized on a gas Weber number $\left(W e_{g}\right)$ versus liquid Reynolds number $\left(R e_{l}\right)$ map, shown in Figure 2. At high $R e_{l}$, the liquid sheet breakup characteristics change based on a modified Ohnesorge number, $O h_{m} \equiv \sqrt{W e_{g}} / R e_{l}$, as follows: (i) at high $O h_{m}$ and high $W e_{g}$, the lobes become thin and puncture, creating holes and bridges. Bridges break as perforations expand and create ligaments. Ligaments then stretch and break into droplets by capillary action; see Figure 3. This domain is indicated as Atomization Domain II in Figure 2, and its mechanism is called LoHBrLiD, based on the cascade of structures that are seen in this domain ( $L o \equiv$ Lobe, $H \equiv$ Hole, $B r \equiv$ Bridge, $L i \equiv$ Ligament, and $D \equiv$ Droplet); (ii) at low $O h_{m}$ and high $R e_{l}$, holes are not seen at early times; instead, many corrugations form on the lobe front edge and then stretch into ligaments. This so called LoCLiD mechanism ( $C \equiv$ Corrugation) occurs in Atomization Domain III (see Figure 2) and results in ligaments and droplets without having the hole and bridge formation steps; see Figure 4. The third mechanism follows a LoLiD process and occurs at low $R e_{l}$ and low $W e_{g}$ (Atomization Domain I in Figure 2), but with some difference in details from the LoCLiD process. The main difference between the two ligament formation mechanisms at high and low $R e_{l}$ 's is that at higher $R e_{l}$ the lobes become corrugated before stretching into ligaments. Hence, each lobe may produce multiple ligaments, which are typically thinner and shorter than those in the lower $R e_{l}$. At low $R e_{l}$, on the other hand, because of the higher viscosity, the entire lobe stretches into one thick, usually long ligament; see Figure 5 . The structures seen in all these breakup mechanisms are sketched in Figure 6. Recent microscopic visualization approaches can deliver high-resolution images, which could be used for the validation of the three main breakup mechanisms introduced. There is also a transitional region in which both lobe/ligament stretching and hole-formation mechanisms are seen simultaneously. The transitional region at low $R e_{l}$ follows a hyperbolic relation, i.e. $W e_{g}=A / R e_{l}$, shown by the dash-dotted line in Figure 2; while at high $R e_{l}$ limit, it follows a parabolic curve, i.e. $W e_{g}=B^{2} R e_{l}^{2}$, shown by the dashed line in Figure 2. The constant $B$ is a critical $O h_{m}$ at high $R e_{l}, B \approx 0.021$. The two borderlines can be combined into a single function with some extra constants for a better fit as follows

$$
W e_{g}=\frac{A}{R e_{l}+\epsilon}+B^{2} \operatorname{Re}_{l}^{2}+C,
$$

where $A, B$ and $C$ are empirical constants, and $\epsilon$ is a small parameter for better curve fitting. As $R e_{l}$ gets very large, we would retain the parabolic function (second term) with the constant $B$ being the product of critical $O h$ and $\sqrt{\hat{\rho}}$. In the limit where $R e_{l}$ gets very small, the hyperbolic function (first term) dominates and gives the asymptote. There are two different characteristic times for the formation of holes and the stretching of lobes and ligaments. At the same $R e_{l}$, as surface tension increases (decreasing $O h_{m}$ and $W e_{g}$ ), the characteristic time for hole formation becomes larger, hence delaying the hole formation. Thus, for lower $O h_{m}$ (or $W e_{g}$ ) most of the earlier ligaments are formed due to direct stretching of the lobes and/or corrugations, while the hole formation is inhibited. On the other hand, at relatively large $\operatorname{Re}_{l}(>3000)$, as the liquid viscosity is increased (decreasing $R e_{l}$ and increasing $\left.O h_{m}\right)$, at the same $W e_{g}$, the ligament-stretching time gets larger. In this case, hole formation prevails compared

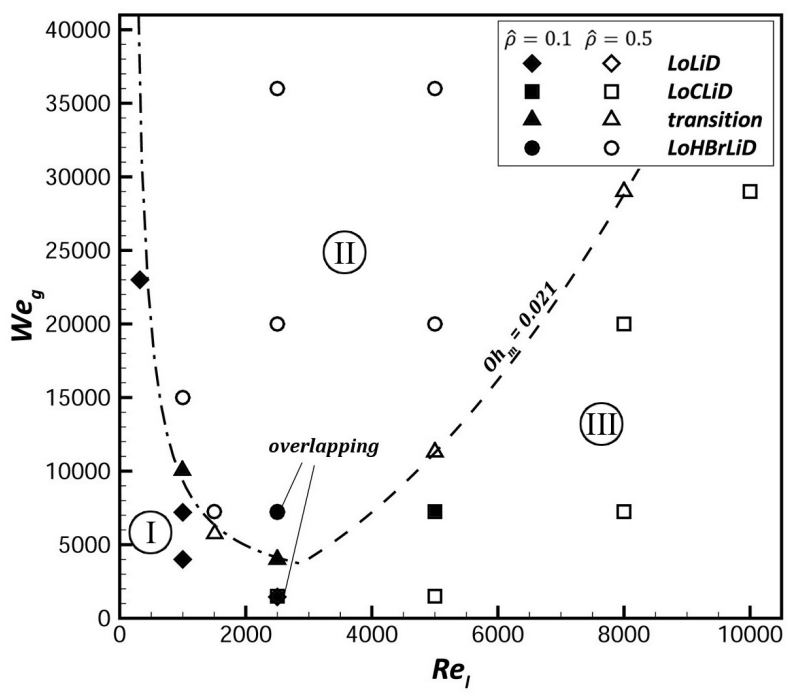

Figure 2. The breakup characteristics based on $W e_{g}$ and $R e_{l}$, showing the LoLiD mechanism denoted by diamonds (Atomization Domain I), the $\mathrm{LoHBrLiD}$ mechanism denoted by circles (Atomization Domain II), the LoCLiD mechanism denoted by squares (Atomization Domain III), and the transitional region denoted by triangles. The $\hat{\rho}=0.1$ cases are shaded. The low and high density ratio cases that overlap at the same point on this diagram have been marked; $-\cdot-\cdot-$, transitional boundary at low $R e_{l}$; and---, transitional boundary at high $R e_{l}$. 


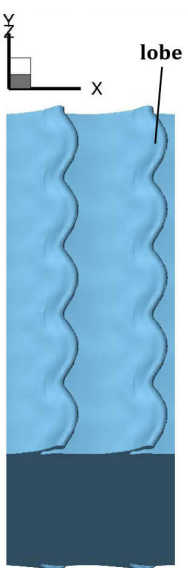

(a)

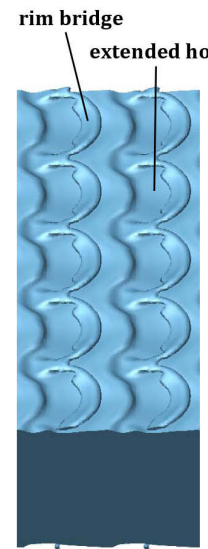

(b)

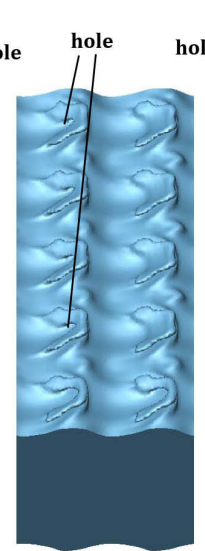

(c)

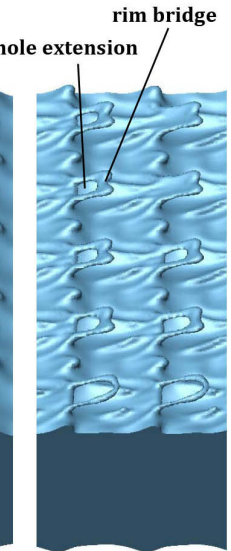

(d)

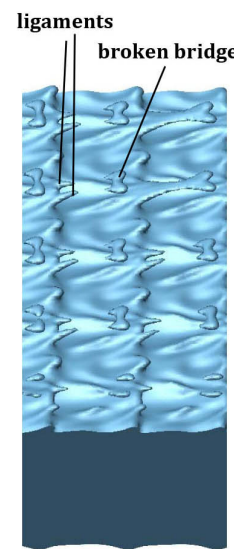

(e)

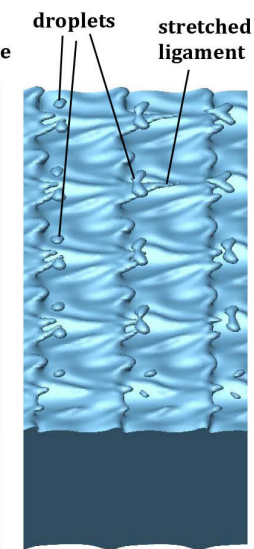

(f)

Figure 3. Liquid surface deformation in the $\mathrm{LoHBr} L i D$ mechanism (Domain II); $R e_{l}=320, W e_{g}=115,000, \hat{\rho}=0.5$, and $\hat{\mu}=0.0022$, at $t=18 \mu \mathrm{s}(a), 22 \mu \mathrm{s}(b), 26 \mu \mathrm{s}(c), 28 \mu \mathrm{s}(d), 30 \mu \mathrm{s}(e)$, and $32 \mu \mathrm{s}(f)$. Gas flows from left to right.

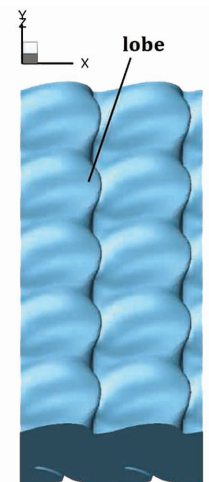

(a)

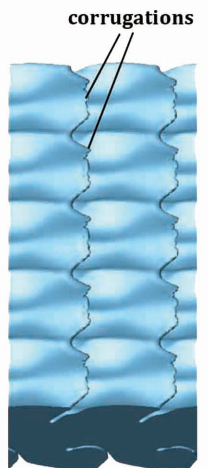

(b)

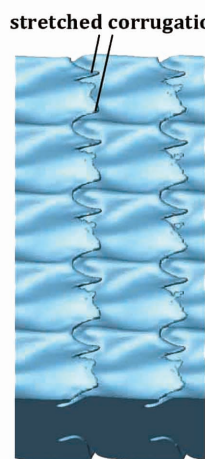

(c)

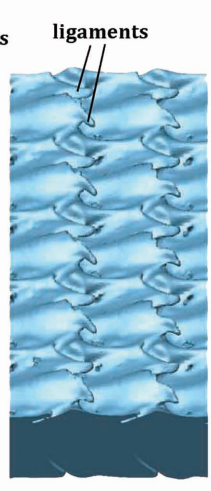

(d)

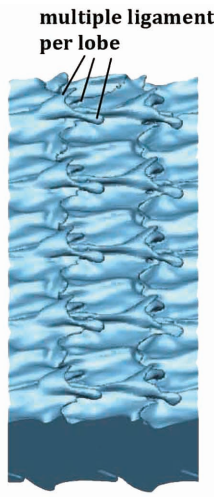

(e)

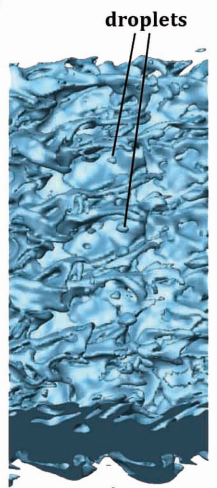

$(f)$

Figure 4. Liquid surface deformation in the $L o C L i D$ mechanism (Domain III); $R e_{l}=5000, W e_{g}=7200, \hat{\rho}=0.5$, and $\hat{\mu}=0.0066$, at $t=44 \mu(a), 48 \mu \mathrm{s}(b), 50 \mu \mathrm{s}(c), 52 \mu \mathrm{s}(d), 56 \mu \mathrm{s}(e)$, and $60 \mu \mathrm{s}(f)$. Gas flows from left to right.

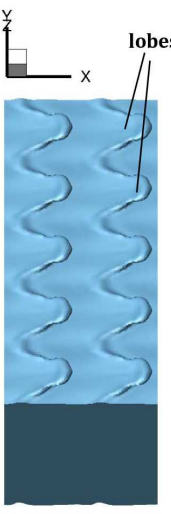

(a)

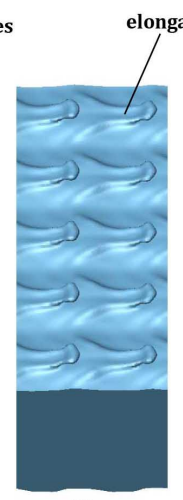

(b)

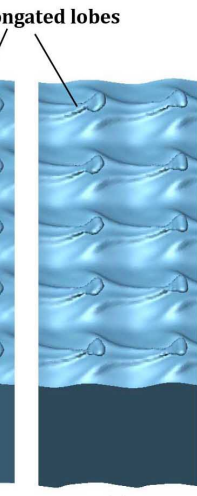

(c)

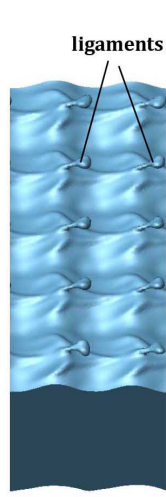

(d)

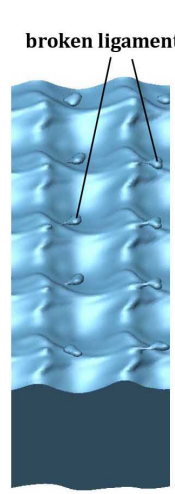

(e)

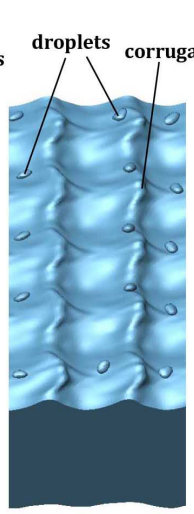

$(f)$ stretching corrugations

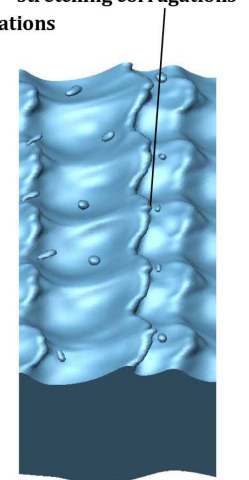

$(g)$

Figure 5. Liquid surface showing the $L o L i D$ mechanism (Domain I) at low $\operatorname{Re}_{l} ; \operatorname{Re}_{l}=320, W e_{g}=23,000, \hat{\rho}=0.1$ and $\hat{\mu}=0.0022$, at $t=26 \mu \mathrm{s}(a), 36 \mu \mathrm{s}(b), 40 \mu \mathrm{s}(c), 44 \mu \mathrm{s}(d), 46 \mu \mathrm{s}(e), 48 \mu \mathrm{s}(f)$, and $52 \mu \mathrm{s}(g)$. Gas flows from left to right.

to the ligament stretching mechanism, resulting in more holes on the liquid lobes. As $W e_{g}$ increases, the time at which the first holes form decreases. This indicates that the hole formation characteristic time should be inversely proportional to $W e_{g}$. At low $R_{l}(<3000)$, the liquid viscosity has an opposite effect on the hole formation and ligament stretching. As shown in Figure 2, near the left boundary, the time scale of the stretching becomes relatively smaller than the hole-formation time scale as $R e_{l}$ is reduced at a constant $W e_{g}$. Hence, there is a move back to ligament stretching from hole formation with decreasing $R e_{l}$ at a fixed $W e_{g}$. Keeping all these effects in mind, the following two nondimensional characteristic times are proposed for these mechanisms:

$$
\frac{U \tau_{h}}{h} \propto \frac{\sigma}{\rho_{g} U^{2} h}\left(1+\frac{k}{R e_{l}}\right)=\frac{1}{W e_{g}}\left(1+\frac{k}{R e_{l}}\right), \quad \frac{U \tau_{s}}{h} \propto \frac{\mu_{l}}{\rho_{l} U h}=\frac{1}{R e_{l}},
$$



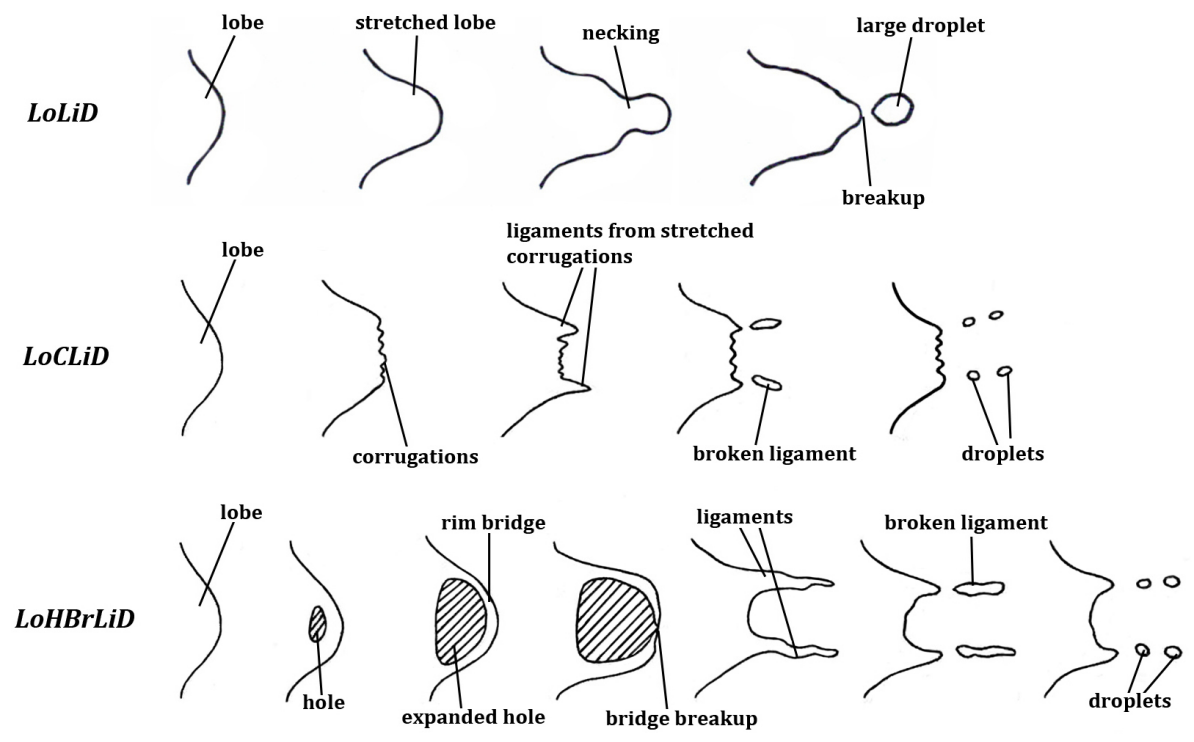

Figure 6. Sketch showing the cascade of structures on a liquid lobe from a top view for the LoLiD (top), LoCLiD (center), and LoH BrLiD (bottom) mechanisms. The gas flows from left to right and time increases to the right.

where $\tau_{h}$ and $\tau_{s}$ are the dimensional characteristic times for hole formation and ligament stretching, respectively, and $k$ is a nondimensional constant.

Combination of the above two equations yields a relation between the two time scales involving $O h_{m}$.

$$
\frac{U \tau_{h}}{h} \propto\left(\frac{U \tau_{s} / h}{O h_{m}}\right)^{2}\left(1+\frac{k}{R e_{l}}\right) .
$$

So, at low $R e_{l}$, the hole formation and the ligament stretching time scales are not related only via $O h_{m} ; R_{l}$ also has a significant role in the breakup mechanism at lower $R e_{l}$. At high $R e_{l}$ however, the term in the second parenthesis in equation (6) approaches unity, and the two time scales relate via $O h_{m}$ only. In the transitional region, near the boundary, where the two characteristic times are of the same order, both hole formation and corrugation stretching appear at different parts of the liquid sheet.

The breakup mechanisms are functions of $R e_{l}$ and $W e_{g}$ only. The qualitative behavior is not much affected by the viscosity ratio (thus the gas $R e$ ); the influence of the density ratio appears only through $W e_{g}$, and the effect of the sheet thickness appears only through $R e_{l}$ and $W e_{g}$.

\section{Vortex dynamics in the LoHBrLiD mechanism}

The vortex structures in the $\mathrm{LoHBrLiD}$ breakup mechanism are sketched in Figure 7. This figure shows the liquid surface (a lobe) and also the qualitative location of the nearby vortices some time after the start of injection. Hairpin vortices form on the braids, between two consecutive $\mathrm{KH}$ vortices, where the maximum strain rate occurs [8]. The hairpins closer to the streamwise $\mathrm{KH}$ wave crest are stretched downstream towards the $\mathrm{KH}$ vortex, and the hairpins

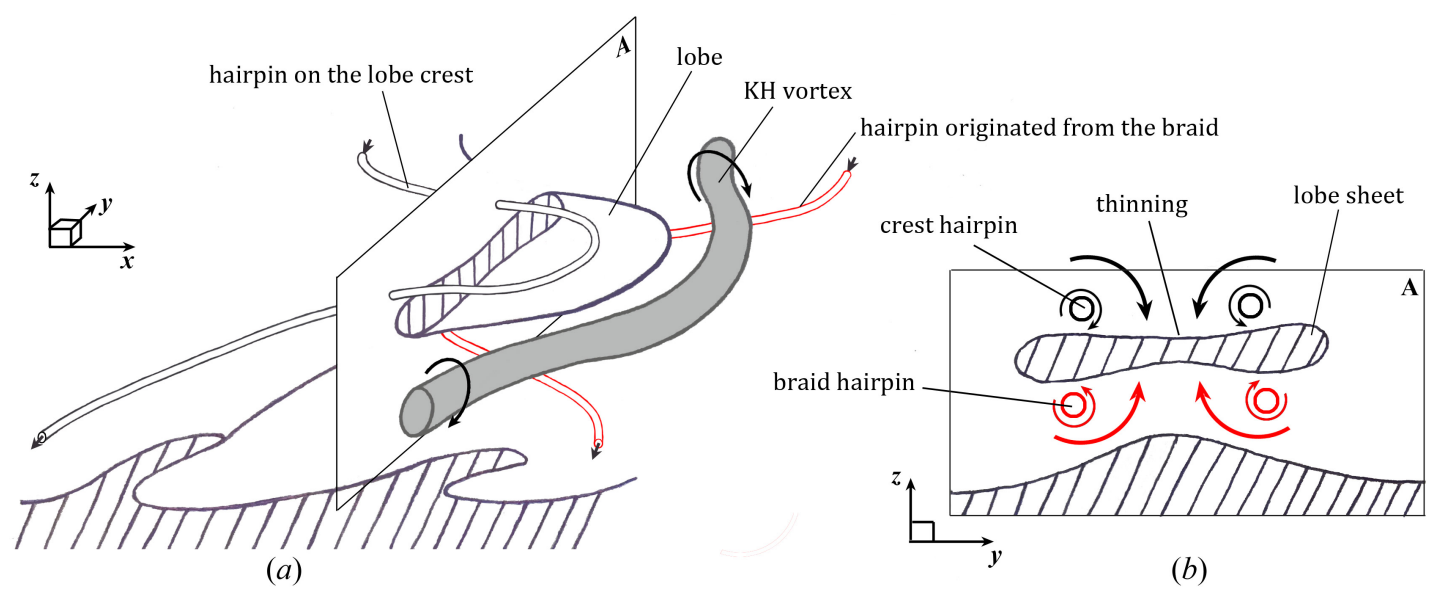

Figure 7. 3D Schematics showing the overlapping of the two hairpin vortices - one from the lobe crest (black tube) and the other from the braid (red tube) $(a)$ - $A$ is the plane in which $(b)$ is drawn; cross-sectional view of the $A$-plane showing the thinning of the lobe sheet due to the hairpin overlapping $(b)$. The vortex schematics are meant to be periodic in $x$ - and $y$-directions. 
near the streamwise trough are stretched upstream due to the pull from the upstream $\mathrm{KH}$ roller. The hairpins that are stretched downstream are rolled over the $\mathrm{KH}$ vortex tube, while the hairpins that are stretched upstream are pulled under the $\mathrm{KH}$ vortex tube.

Later, the $\mathrm{KH}$ vortices deflect more under the influence of the hairpin vortex filaments. The $\mathrm{KH}$ vortex pulls the lobes over itself and lies underneath the surface of the lobe at later times. Pulling of the upstream and downstream hairpins from the outer and inner sides of the interface by the $\mathrm{KH}$ roller causes these two hairpin groups to collect together and overlap on the outer side of the lobe, i.e. on the streamwise wave crest, and its inner side; i.e. on the streamwise wave trough. As shown in Figure 7 and also described by Jarrahbashi et al. [5], the liquid sheet between a pair of these overlapping hairpins becomes thinner. Figure 7(a) schematically depicts two neighboring hairpin vortices in the liquid-gas interface region - one originating from the lobe crest and stretching downstream (shown by the slender black tube) and the other originating from the braid and stretching upstream (shown by the red tube). The $\mathrm{KH}$ vortex is shown by the thicker gray tube in this figure. Figure $7(b)$ shows a cross-sectional view of the vortex structure along with the liquid sheet located between the two hairpin vortices on the $A$-plane of Figure $7(a)$. The induced velocities of the overlapping hairpin vortices (see the qualitative streamlines shown by the black and red arrows in Figure $7 b$ ) push the top surface of the liquid lobe downward and the bottom surface upward, causing the liquid lobe to become thinner in the middle and thus vulnerable to puncture at that region.

Whether the liquid sheet subject to these conditions punctures or not depends on other flow conditions, particularly the surface tension. At high $W e_{g}$ (high $O h_{m}$ ), the hole formation prevails and occurs at the predicted locations. As these overlapping hairpin filaments continue to stretch, the holes stretch and expand with them, creating even larger perforations and thinner bridges. If $W e_{g}$ is not large enough, the liquid sheet in the overlapping region can recover instead. In this case, hole formation is inhibited, and the lobes stretch directly, but more slowly, into ligaments via LoLiD or LoCLiD mechanisms (Domains I and III), as will be discussed in the next sections.

\section{Vortex dynamics in the LoCLiD mechanism}

The role of vortices on the liquid surface deformation at high $R e_{l}$ and low $W e_{g}$ is summarized schematically in Figure 8. At early times $t_{1}$, the spanwise vortices on the braid deflect due to the pull from the neighboring $\mathrm{KH}$ rollers in both the upstream and downstream directions, creating the hairpin vortex structures with a wavelength equal to the spanwise perturbation wavelength. The deflected hairpin filaments form the lobes as they are stretched by the $\mathrm{KH}$ roller. So far, the process is similar to the $\mathrm{LoHBrLiD}$ mechanism.

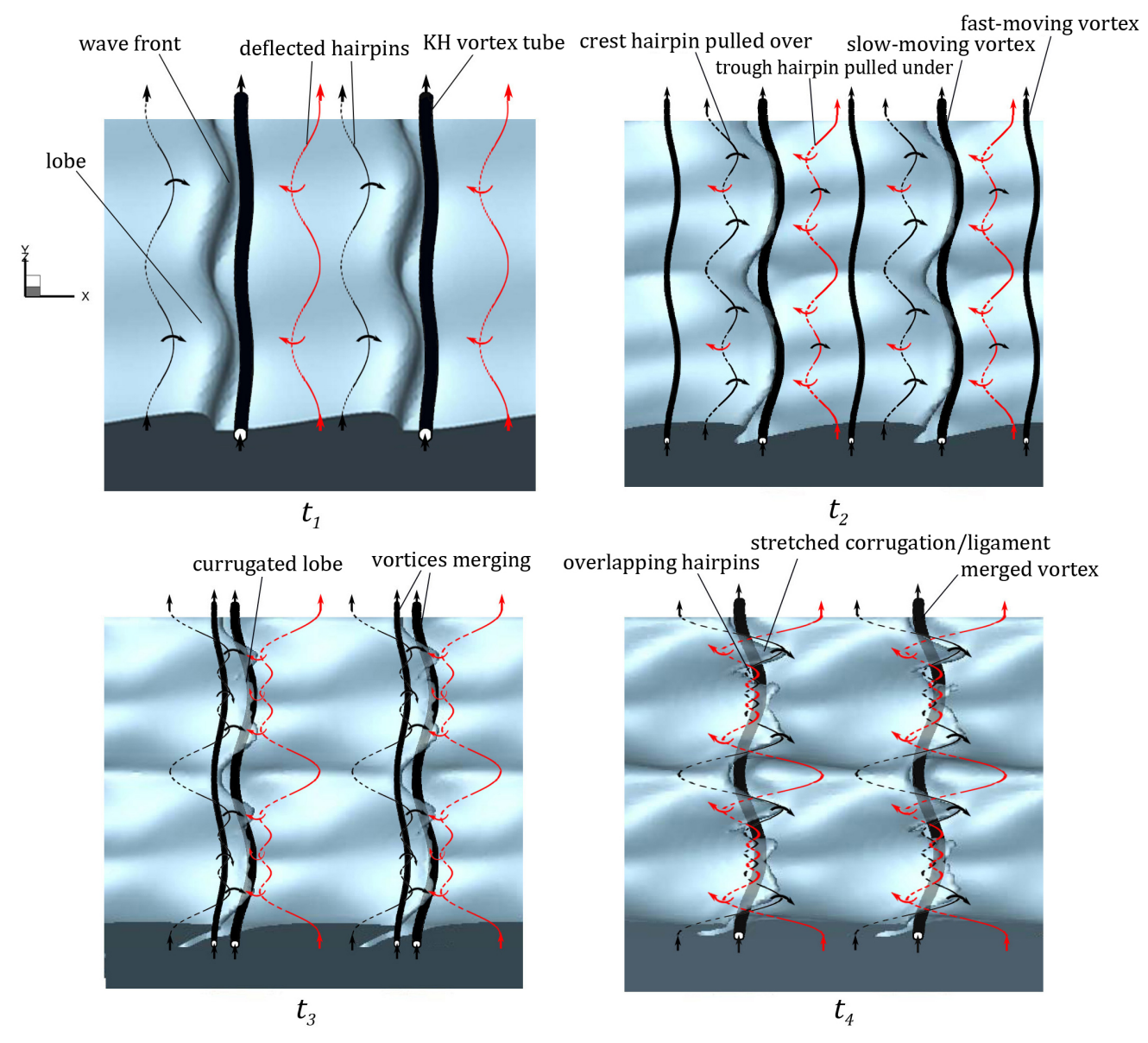

Figure 8. Schematics of the $\mathrm{LoCLiD}$ mechanism at four consecutive times. The liquid surface is shown in blue, and the the $\mathrm{KH}$ vortex in black. The red and black lines are the hairpin vortices near the trough and crest of the KH wave, respectively. The solid and dashed lines show where the hairpins are stretched upstream and inward, or downstream and outward, respectively. 
At high $R e_{l}$, the inertial effects dominate the viscous forces. Consequently, the higher velocity of the gas phase compared to the liquid phase causes the $\mathrm{KH}$ roller to split into two eddies at $t_{2}$, as shown in Figure 8 . The low liquid viscosity does not allow sufficient resistance to keep the $\mathrm{KH}$ vortex bound near the interface; hence, the outer part of the $\mathrm{KH}$ roller, which resides in the fast-moving gas layer, separates from the part of the $\mathrm{KH}$ vortex that is closer to the liquid surface at lower speed.

As demonstrated in Figure 8, the part of the $\mathrm{KH}$ vortex that resides outside of the liquid advects faster than the slowmoving eddy in the liquid. The slow-moving eddy advects with the interface velocity and remains stationary relative to the liquid surface. This vortex separation has two significant effects. (i) The slow-moving eddy downstream of the $\mathrm{KH}$ wave is not strong enough to curl the $\mathrm{KH}$ wave and pull the lobe downstream over itself. Consequently, the outer hairpin filaments on the lobe do not overlap with the inner braid hairpins as in the $\operatorname{LoHBrLiD}$ process, and hole formation at early times is inhibited. (ii) The fast-moving eddy gets closer to the downstream hairpins as it moves farther from the upstream hairpins near the trough. The hairpin filaments become less orderly due to the time-variant pull from this fast-moving eddy in opposite directions. As the fast-moving vortex passes over the trough hairpin (the red line), it pulls the trough hairpin in the downstream direction and creates a new bend on the hairpin, as shown in Figure 8 at $t_{2}$. Similarly, the crest hairpin filament (the black line) is pulled in the upstream direction by the fast-moving vortex tube, causing the hairpin vortices to undergo more undulations with smaller wavelengths, and also prevents further streamwise stretch in the original hairpins. Consequently, the lobes are less stretched and more blunt compared to the LoHBrLiD mechanism.

When the fast-moving eddy passes over the crest hairpin (black hairpin) at $t_{3}$, it creates yet another turn in the hairpin vortex, as shown in Figure 8. The liquid surface approximately follows the hairpin structures with some delay at this high $R e_{l}$ range - as the vortex lines are nearly material lines. Because of these shorter hairpin wavelengths, corrugations with length scales comparable to the hairpin wavelengths are formed on the front-most edge of the lobes, as shown in Figure 8 at $t_{3}$. Both experimental observations [8,14] and numerical simulations [20,21] show that the number of corrugations (lobes) increases by increasing the jet Reynolds number.

Upon creation of a stronger eddy downstream of the $\mathrm{KH}$ waves at $t_{4}$ - after amalgamation of the fast- and slowmoving eddies - the new KH roller (now a thicker tube) has enough strength to stretch the hairpin vortices. The hairpin filaments on the outer and inner sides of the lobe overlap as illustrated in Figure 8 at $t_{4}$. In this figure, the dashed lines represent the hairpins stretching upstream and under the liquid lobes, i.e. inner surface of the gas lobe, while the solid lines are parts of the hairpin filament that are stretched downstream on the inner side of the liquid lobe; i.e outer surface of the gas lobe.

The flow induced by the hairpin vortices creates undulations on both the bottom surface of the lobe and the trough surface. The gap filled by the gas layer gets closed, i.e. the lobe collapses on the jet core, as the bottom surface of the liquid lobe descends and the trough surface ascends. The counter-rotating pairs of streamwise vortices on the liquid lobe create the ligaments on the two sides of the lobe at $t_{4}$ (see Figure 8 ). The corrugations also stretch into ligaments but at a slower growth rate. The ligaments stretch downstream and break into droplets as they undergo capillary instabilities later. In the meantime, the eddies separate from the interface and advect outward into the gas phase.

\section{Vortex dynamics in the LoLiD mechanism}

Similar to the previous cases, the vortex field starts with a large $\mathrm{KH}$ roller downstream of the $\mathrm{KH}$ wave, hairpin filaments on the braid, and a stronger hairpin filament on the $\mathrm{KH}$ wave crest. Later, the entire $\mathrm{KH}$ roller departs from the liquid surface and moves downstream into the gas zone. The $\mathrm{KH}$ roller gains higher velocity as it moves away from the interface, while the rest of the hairpin vortices are almost stationary with respect to the interface.

While the $\mathrm{KH}$ roller reaches the neighboring downstream crest hairpin filament, the braid hairpins overlap with the crest hairpins constraining the lobe sheet in between. So far, the vortex dynamics manifest the conditions required for both hole formation and corrugation formation. However, none of these structures are seen on the lobe. The

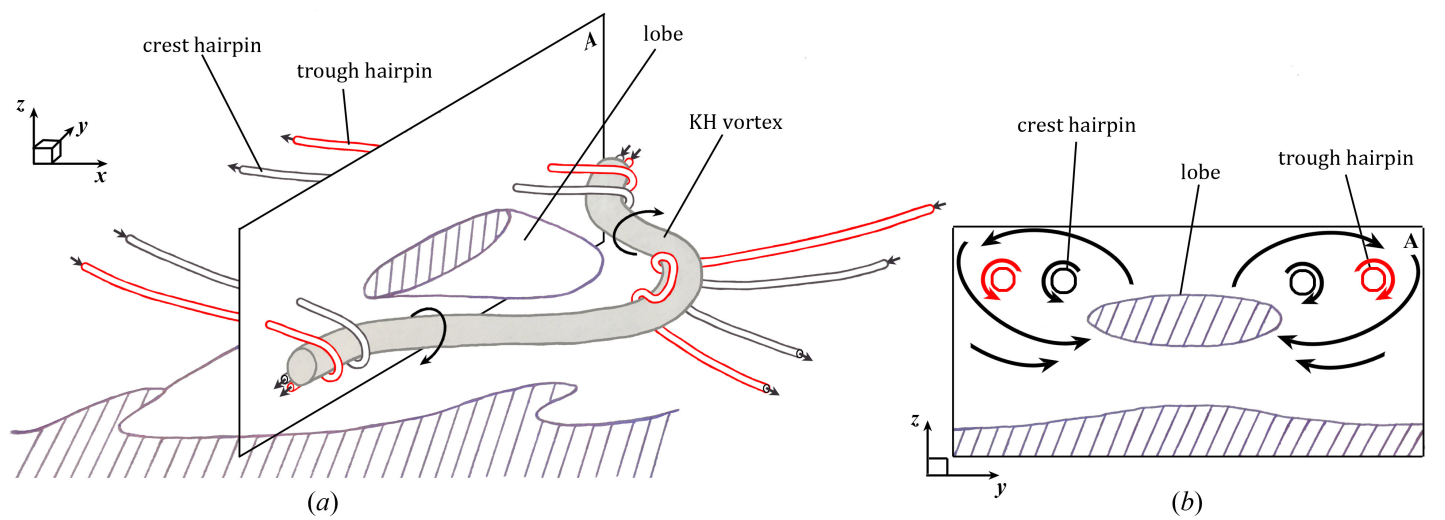

Figure 9. 3D Schematics showing the vortex structures in the LoLiD mechanism of Domain I $(a), A$ is the plane in which $(b)$ is drawn; cross-sectional view of the $A$-plane showing the spanwise squeezing of the lobe by the hairpin vortices $(b)$. The vortex schematics are meant to be periodic in $x$ - and $y$-directions. 
inhibition of the hole formation is due to the high surface tension. In such low $W e_{g}$, the inertial and viscous forces are not strong enough to overcome the surface tension force to thin and stretch the lobe; hence, the lobe perforation is inhibited. Also, because of the high liquid viscosity at such low $R e_{l}$, the liquid surface response to the vortex structures is retarded (vortex lines and material lines are not identical). Hence, the lobe edges are not corrugated following the hairpin filaments as with higher $R e_{l}$. Moreover, the $\mathrm{KH}$ roller is more distant from the interface in this case compared to the LoCLiD mechanism, which means that it has a much weaker influence on the hairpin filaments, resulting in fewer bends and less undulation on the hairpins.

The schematics of the vortex structures in the LoLiD mechanism of Domain I are illustrated in Figure 9. The $\mathrm{KH}$ vortex has a higher undulation in this domain compared to the other two domains and is also farther away from the interface in the gas zone. Because of the high strain rate at the braid, the hairpin vortices become streamwise near the braid region in both spanwise crest and trough. Two pairs of counter-rotating hairpins - one on the lobe crest (shown by the black tube) and the other on the trough (shown by the red tube) - stretch and wrap around the KH vortex; see Figure $9(a)$. As shown in the cross-sectional view of the $A$-plane passing through the lobe (Figure $9 b$ ), both the black and the red hairpins are located slightly above and on the sides of the lobe at this moment. While the flow induced by the $\mathrm{KH}$ vortex creates a streamwise flow on the two sides of the lobe, the gas flow induced by these two counter-rotating hairpins (shown by the curly arrows is Figure $9 b$ ) generates a spanwise flow towards the lobe. Consequently, the lobe is both squeezed in the spanwise direction - via the hairpins effects - and stretched in the streamwise direction by the induced flow of the $\mathrm{KH}$ vortex. The gas flow also lifts the lobe in the normal direction. The flow induced by the streamwise hairpins transforms the lobe into a thick ligament as the strong shear near the lobe sides deforms the liquid surface.

\section{Conclusions}

The temporal development of surface waves and their breakup into droplets are studied numerically. Three main breakup mechanisms are identified. The breakup characteristics are well categorized on a parameter space of gas Weber number $\left(W e_{g}\right)$ versus liquid Reynolds number $\left(R e_{l}\right)$. The atomization regime is now separated as three sub-domains.

The vortex dynamics is able to explain the hairpins formation, and the interaction between the hairpin vortices and the $\mathrm{KH}$ vortex explains the perforation of the lobes at moderate $R e_{l}$ and high $W e_{g}$, which is attributed to the overlapping of the two pairs of hairpin vortices on top and bottom of the lobe. The formation of corrugations on the lobe front edge at high $R e_{l}$ is also explained by the structure of the hairpins and the splitting of the $\mathrm{KH}$ vortex. At low $R e_{l}$ and low $W e_{g}$, on the other hand, the lobe perforation and corrugation formation are inhibited due to high surface tension and high liquid viscosity. The hairpin vortices stretch in the normal direction while wrapping around the $\mathrm{KH}$ vortex. The induced gas flow squeezes the lobe from the sides and forms a thick and long ligament.

\section{References}

[1] Shinjo, J., and Umemura, A., 2010, International Journal of Multiphase Flow, 36, pp. 513-532.

[2] Desjardins, O., and Pitsch, H., 2010, Atomization and Sprays, 20 (4), pp. 311-336.

[3] Herrmann, M., 2011, Atomization and Sprays, 21 (4), pp. 283-301.

[4] Jarrahbashi, D., and Sirignano, W. A., 2014, Physics of Fluids, 26 (10), p. 73.

[5] Jarrahbashi, D., Sirignano, W. A., Popov, P., and Hussain, F., 2016, Journal of Fluid Mechanics, 792, pp. 186-231.

[6] Bernal, L. P., and Roshko, A., 1986, Journal of Fluid Mechanics, 170, pp. 499-525.

[7] Pope, S. B., 1978, AIAA Journal, 16 (3), pp. 279-281.

[8] Lasheras, J. C., and Choi, H., 1988, Journal of Fluid Mechanics, 189, pp. 53-86.

[9] Schowalter, D. G., Van Atta, C. W., and Lasheras, J. C., 1994, Meccanica, 29 (4), pp. 361-371.

[10] Widnall, S. E., and Sullivan, J. P., 1973, Proceedings of the Royal Society of London A: Mathematical, Physical and Engineering Sciences, 332 (1590), pp. 335-353.

[11] Widnall, S. E., Bliss, D. B., and Tsai, C. Y., 1974, Journal of Fluid Mechanics, 66 (1), pp. 35-47.

[12] Breidenthal, R. E., 1981, Journal of Fluid Mechanics, 109, pp. 1-24.

[13] Jimenez, J., 1983, Journal of Fluid Mechanics, 132, pp. 319-336.

[14] Liepmann, D., and Gharib, M., 1992, Journal of Fluid Mechanics, 245, pp. 643-668.

[15] Ashurst, W. T., and Meiburg, E., 1988, Journal of Fluid Mechanics, 189, pp. 87-116.

[16] Martin, J. E., and Meiburg, E., 1991, Journal of Fluid Mechanics, 230, pp. 271-318.

[17] Comte, P., Lesieur, M., and Lamballais, E., 1992, Physics of Fluids A: Fluid Dynamics, 4 (12), pp. 2761-2778.

[18] Collis, S. S., Lele, S. K., Moser, R. D., and Rogers, M. M., 1994, Physics of Fluids, 6 (1), pp. 381-396.

[19] Schoppa, W., Hussain, F., and Metcalfe, R. W., 1995, Journal of Fluid Mechanics, 298, pp. 23-80.

[20] Brancher, P., Chomaz, J. M., and Huerre, P., 1993, Physics of Fluids, 6 (5), pp. 1768-1774.

[21] Danaila, I., Dusek, J., and Anselmet, F., 1997, Physics of Fluids, 9 (11), pp. 3323-3342.

[22] Zandian, A., Sirignano, W. A., and Hussain, F., 2016, 54th AIAA Aerospace Sciences Meeting, p. 1593.

[23] Zhao, H. K., Chan, B., Merriman, B., and Osher, S., 1996, Journal of computational physics, 127 (1), pp. 179-195.

[24] Sussman, M., Fatemi, E., Smereka, P., and Osher, S., 1998, Computers and Fluids, 27 (5), pp. 663-680.

[25] Osher, S., and Fedkiw, R. P., 2001, Journal of computational physics, 169 (2), pp. 463-502.

[26] Hirt, C. W. and Nichols, B. D., 1981, Journal of computational physics, 39 (1), pp. 201-225.

[27] Jeong, J., and Hussain, F., 1995, Journal of Fluid Mechanics, 285, pp. 69-94. 